\title{
Desempenho de ferramentas de diamante CVD micro/nanocristalino em torneamento de ligas de alumínio 7075
}

\author{
Performance of micro/nanocrystalline CVD diamond tools in turning of 7075 \\ aluminum alloy
}

Argemiro Pentian Junior ${ }^{* *}$, José Vieira ${ }^{2}$, Vladimir J. Trava Airoldi², João Roberto Moro

\section{RESUMO}

Este trabalho investigou o desempenho de ferramentas de usinagem de carbeto de tungstênio na matriz de cobalto (WC-Co) com deposição de filmes de diamante CVD micro e nanocristalino em liga de alumínio 7075, observando-se a rugosidade da liga usinada e variando os parâmetros de velocidade de corte e o avanço. Para tanto, foram preparados corpos de prova na liga de alumínio 7075 e utilizados três tipos de insertos: um sem revestimento, um com revestimento monocamada microcristalino e um com revestimento nanocristalino. Foram realizados estudos de composição e avaliação da superfície dos insertos antes e após os experimentos, obtendo-se resultados sobre o desgaste de cada inserto usado e comparando-se as rugosidades entre os corpos de prova usinados. Em todos os casos, o aumento do avanço resultou em aumento da rugosidade aritmética, apresentando a ferramenta com revestimento nanocristalino um aumento mais significativo. Comparando os insertos entre si, observa-se que o revestimento microcristalino apresenta variação de rugosidade maior do que nanocristalino, tendo performance mais estável em todas as condições com valores entre 0,4 e 0,6 $\mu \mathrm{m}$, estando o revestimento nanocristalino mais estável para velocidades maiores (acima de 500m. $\mathrm{min}^{-1}$ ) com rugosidade estável em 0,5 $\mu \mathrm{m}$.

Palavras-chave: Diamante, Alumínio, Usinagem, WC-Co, CVD

\begin{abstract}
This paper investigated the performance of tungsten carbide machining tools in the cobalt matrix (WC-Co) with deposition of micro and nanocrystalline CVD diamond films in 7075 aluminum alloy, observing the roughness of the machined bond by varying the cutting speed parameters and the feed. For this purpose, specimens were prepared in 7075 aluminum alloy and three types of inserts were used: one without coating, one with microcrystalline monolayer coating and one with nanocrystalline coating. Composition studies and evaluation of the insert surface before and after the experiments were performed, obtaining results on the wear of each insert used and comparing the roughness between the machined specimens. In all cases, the increase in feed resulted in increased arithmetic roughness, with the tool with nanocrystalline coating showing a more significant increase. Comparing the inserts, it was observed that the microcrystalline coating presents a roughness variation greater than the nanocrystalline coating, having a more stable performance in all conditions with values between 0.4 and $0.6 \mu \mathrm{m}$, the nanocrystalline coating being more stable for higher speeds (above $500 \mathrm{~m} \cdot \mathrm{min}^{-1}$ ) with a stable roughness of $0.5 \mu \mathrm{m}$.
\end{abstract}

Keywords: Diamond, Aluminum, Machining, WC-Co, CVD

1.Instituto Federal de Educação Ciência e Tecnologia de São Paulo - São José dos Campos (SP), Brasil. 2.Instituto Nacional de Pesquisas Espaciais - São José dos Campos (SP), Brasil.

Autor correspondente: junior.pentian@outlook.com

Recebido: 03 Ago. 2019 Aprovado: 27 Ago. 2019 


\section{INTRODUÇÃO}

O alumínio, desde sua obtenção comercialmente viável em 1886, é uma das matérias-primas mais utilizadas pela indústria metalmecânica. Seu uso é interessante por possuir propriedades como baixa densidade, boa condutividade, resistência e boa usinabilidade, sendo bem visto pelos mercados nacional e internacional, além de possuir alta reciclabilidade, fator importante em tempos de preocupação ambiental e economia de energia.

Ligas da série $7 \mathrm{xxx}$, como a 7050 e a 7475 , são utilizadas na indústria aeronáutica em vários modelos de aviões, como B787, Airbus A380, F35 e Typhoon ${ }^{1}$, em praticamente todas as partes do avião, desde a asa até a fuselagem, sendo responsáveis por $80 \%$ do peso da aeronave ${ }^{2}$ despertando especial interesse por seu bom comportamento em situações de fadiga e corrosão.

Com a demanda industrial dessa liga, as empresas necessitam de maiores informações sobre seu comportamento durante o processo de fabricação visando obter resultados otimizados tanto do ponto de vista produtivo quanto comercial.

Entre os diversos processos de fabricação, a usinagem é um dos mais utilizados. Nesse campo, a demanda por ferramentas de metal duro com melhor desempenho a um custo razoável e a utilização de alta velocidade de usinagem conduzem à procura de materiais novos e mais avançados com melhores características de desgaste ${ }^{3}$.

O aumento da velocidade de corte implica aumento da taxa de remoção, reduzindo o custo de material removido, mas aumenta o uso de fluido refrigerante e reduz a vida da ferramenta, elevando os custos do trabalho. A substituição das ferramentas e os cuidados necessários com a manutenção de fluidos refrigerantes por vezes são maiores que o valor da ferramenta ${ }^{4,5}$. Com isso, a busca por maiores taxas de produção está diretamente relacionada com a pesquisa de materiais para ferramentas não só mais resistentes ao desgaste, mas ainda com a finalidade de diminuir o uso de lubrificantes.

Pode-se destacar dentre essas ferramentas as de metal duro, diamante como carbono (DLC do inglês diamond-like carbon) e carbeto cementado. O carbeto cementado mais importante é carbeto de tungstênio na matriz de cobalto (WC-Co), um ligante metálico dúctil utilizado em proporções de 4 a $20 \%{ }^{5,6}$.

A deposição de filmes de diamante obtido por deposição química a partir da fase vapor (CVD, do inglês chemical vapor deposition) em carbetos de tungstênio cementados tem grande aplicação nas ferramentas de corte ${ }^{7}$ e se justifica pelo baixo coeficiente de atrito, permitindo a redução da força de corte durante o processo de usinagem, facilitando a remoção de cavacos, um benefício tanto para o aprimoramento do desempenho de corte quanto para o prolongamento da vida da ferramenta ${ }^{8}$.

\section{MATERIAIS E MÉTODOS}

Os testes foram realizados em liga de alumínio 7075-T6. A composição química típica dessa liga é apresentada na Tabela 1.

Tabela 1: Especificações de composição química da liga de alumínio 7075-T6 (porcentagem em peso) e dureza.

\begin{tabular}{|c|c|}
\hline Si & $0,110 \%$ \\
\hline $\mathbf{Z n}$ & $5,800 \%$ \\
\hline $\mathrm{Fe}$ & $0,210 \%$ \\
\hline $\mathrm{Ti}$ & $0,036 \%$ \\
\hline $\mathrm{Cu}$ & $1,400 \%$ \\
\hline $\mathrm{Cr}$ & $0,210 \%$ \\
\hline $\mathrm{Mn}$ & $0,190 \%$ \\
\hline $\mathrm{Zr}$ & $0,022 \%$ \\
\hline $\mathrm{Mg}$ & $2,400 \%$ \\
\hline Dureza & $177 \mathrm{HBW}$ \\
\hline
\end{tabular}

Fonte: Coppermetal Aços e Metais ${ }^{9}$

Especificação do material:

- Liga de alumínio AA7075-T6 Ø 2" × 1.000 mm;

- Resistência à tração: 599,71 MPa;

- Limite de escoamento: 542,46 MPa;

- Alongamento: 8,50\%

- T6: Solubilizado e envelhecido artificialmente. ${ }^{9}$

Os corpos de prova foram preparados com $\varnothing 50 \mathrm{~mm} \times 120 \mathrm{~mm}$ de comprimento (Fig. 1).

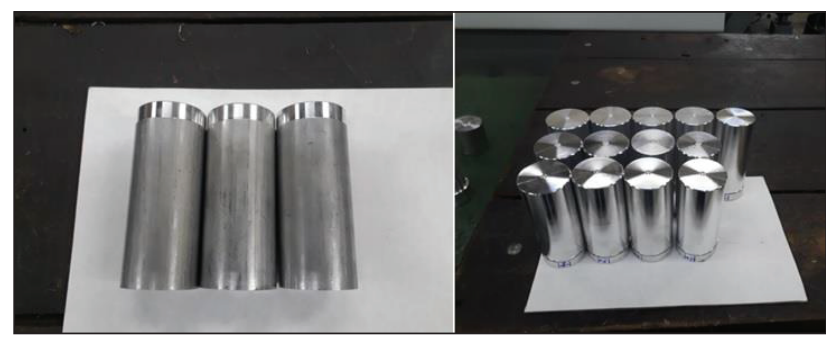

Figura 1: Corpos de prova antes e depois da preparação.

Os insertos utilizados são pastilhas de ferramentas de corte de metal duro comercialmente disponíveis pela empresa Brassinter, modelo TNMA 160408 e possuem as características descritas na Fig. 2.

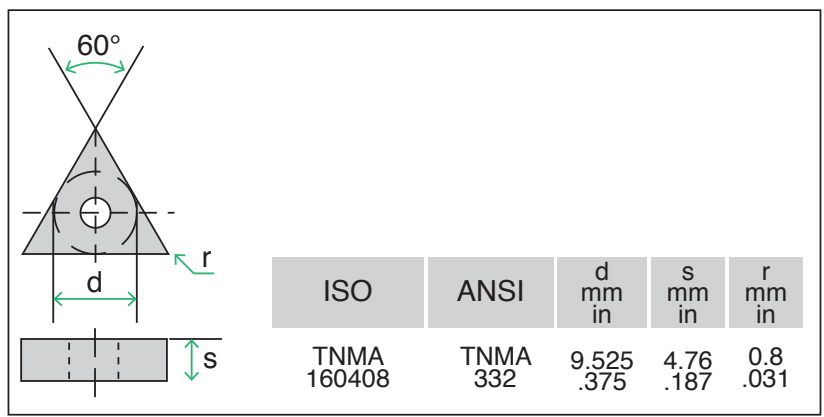

Figura 2: Características das ferramentas do experimento. 
Os testes de torneamento foram realizados em um torno $\mathrm{CNC}$ TND200, fabricante ERGOMAT; rotação máxima: $5.000 \mathrm{rpm}$; potência: $11 \mathrm{~kW}$; placa e contraponto hidráulicos pertencentes ao Laboratório de Usinagem do Instituto Federal de São Paulo (IFSP), Câmpus São Paulo.

Os parâmetros do experimento utilizados foram: velocidade de corte: 424-600 m. $\mathrm{min}^{-1}$; avanço: 0,06-0,15 mm.rev ${ }^{-1}$; profundidade de corte: $1 \mathrm{~mm}$.

\section{Procedimento de tratamento de superfície}

A preparação da superfície tem um papel destacado no controle da nucleação, sendo importante na obtenção de filmes de diamante de alta aderência e com qualidade. $\mathrm{O}$ aumento na densidade de nucleação pode melhorar a homogeneidade dos filmes, aumentando a adesão entre filme e substrato ${ }^{10}$.

A existência de cobalto na superfície dos substratos não é desejável, pois é um metal de transição e, durante o depósito de diamante CVD, influencia negativamente na aderência do filme ${ }^{11}$. Portanto a estratégia utilizada foi a preparação da superfície em três etapas conforme descritos na Tabela 2.

A nucleação de diamante CVD sobre os substratos foi conduzida através da exposição dos substratos prontos às condições de crescimento em reator de filamento quente (HFCVD, do inglês hot filament chemical vapor deposition) com os parâmetros expostos na Tabela 3 .

\section{RESULTADOS E DISCUSSÕES Comportamento da rugosidade em função da velocidade de corte}

Os experimentos foram realizados com as velocidades de corte $\left(\mathrm{Vc}=600 ; 500 \mathrm{e} 424 \mathrm{~m} \cdot \mathrm{min}^{-1}\right)$, avanços $(0.06,0.08,0.1,0.12 \mathrm{e} 0.15 \mathrm{~mm})$ e profundidade de corte $(1 \mathrm{~mm})$ definidos para todos os insertos estudados. A leitura dos valores obtidos no experimento foi auferida em três diferentes posições do diâmetro usinado, tomando como referências as castanhas da placa que estão $120^{\circ}$ distantes entre si, usando a média dos valores.

A Fig. 3 mostra o resultado da variação da rugosidade aritmética obtida com esses parâmetros aplicados em um inserto sem revestimento.

Observando o gráfico da Fig. 4, pode-se verificar um comportamento similar a todas as velocidades: instabilidade nos valores das medições de rugosidade nos avanços de 0,06 e $0,08 \mathrm{~mm}$; instabilidade que se alonga entre 0,10 e $0,12 \mathrm{~mm}$, tornando-se agressiva em $0,15 \mathrm{~mm}$; até $0,12 \mathrm{~mm}$, essa instabilidade se mantém dentro dos valores de 0,4 e $0,8 \mu \mathrm{m}$, com melhor performance em $500 \mathrm{~m} \cdot \mathrm{min}^{-1}$ e pior em $424 \mathrm{~m} \cdot \mathrm{min}^{-1}$.

Em condições de baixas velocidades de corte, ocorre a formação de aresta postiça na ferramenta. Durante a usinagem, a aresta postiça se quebra ciclicamente e é removida da área de

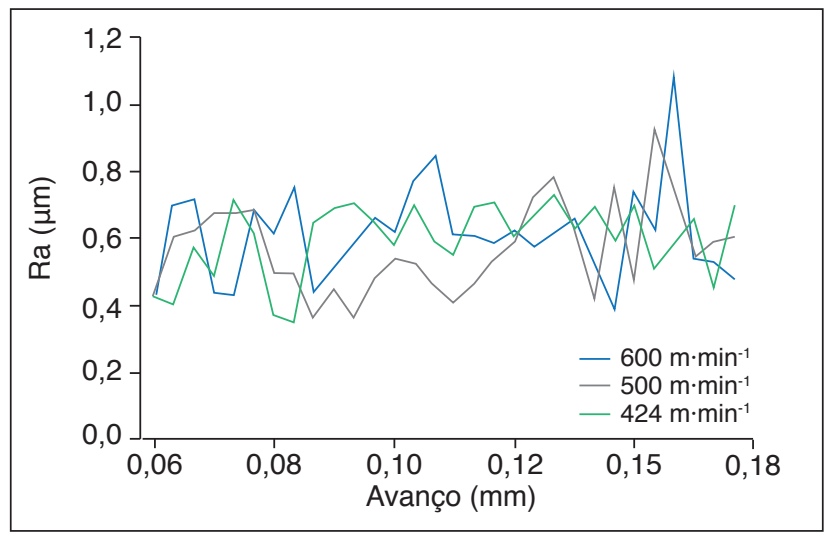

Figura 3: Comparativo de rugosidade entre velocidades sem revestimento.

Tabela 2: Condições de pré-tratamento dos substratos.

\begin{tabular}{c|c} 
Pré-tratamento & Condiçães $^{12}$ \\
\hline 1 & Passo 1: 10 min em solução Murakami $\left(\mathrm{K}_{3} \mathrm{Fe}(\mathrm{CN})_{6}: \mathrm{KOH}: \mathrm{H}_{2} \mathrm{O}=1: 1: 10\right)$ \\
\hline 2 & Passo 2: 5 min em água régia $\left(\mathrm{HNO}_{3}: \mathrm{HCl}=1: 3\right)$ \\
\hline 3 & Passo 1: 10 min em solução Murakami $\left(\mathrm{K}_{3} \mathrm{Fe}(\mathrm{CN})_{6}: \mathrm{KOH}: \mathrm{H}_{2} \mathrm{O}=1: 1: 10\right)$ \\
& Passo $2: 5$ min em água régia diluída $\left(\mathrm{HNO}_{3}: \mathrm{HCl}_{2}: \mathrm{H}_{2} \mathrm{O}=1: 1: 1\right)$ \\
& Passo 1: 10 min em solução Murakami $\left(\mathrm{K}_{3} \mathrm{Fe}\left(\mathrm{CN}_{6}: \mathrm{KOH}_{2}: \mathrm{H}_{2} \mathrm{O}=1: 1: 10\right)\right.$ \\
\hline
\end{tabular}

Tabela 3: Condições de deposição usadas para crescimento dos filmes de diamante ${ }^{13}$.

\begin{tabular}{|c|c|c|}
\hline Condições & Nano & Micro \\
\hline Distância entre filamento e substrato $(\mathrm{mm})$ & 5 & 850 \\
\hline Temperatura do substrato $\left({ }^{\circ} \mathrm{C}\right)$ & 750 & 2 \\
\hline Taxa de fluxo $\mathrm{CH}_{4}(\mathrm{sccm})$ & 6 & 98 \\
\hline Taxa de fluxo $\mathrm{H}_{2}(\mathrm{sccm})$ & 94 & 50 \\
\hline Pressão $($ Torr $)$ & 30 & Murakami + água régia \\
\hline Pré-tratamento do substrato & Murakami + água régia & 120 \\
\hline Tempo de deposição $(\mathrm{min})$ & 240 & $\sim 1$ \\
\hline Taxa de Crescimento $\left(\mu \mathrm{m} \cdot \mathrm{h}^{-1}\right)$ & $\sim 0,2$ & \\
\hline
\end{tabular}


$\operatorname{corte}^{14}$. Esse fenômeno afeta a superfície de saída de cavacos da ferramenta, alterando a geometria de corte e, consequentemente, o acabamento superficial da peça é prejudicado.

Para o inserto com revestimento nanocristalino demonstrado na Fig. 4, observa-se nas velocidades de 600 e $500 \mathrm{~m} \cdot \mathrm{min}^{-1} \mathrm{um}$ comportamento muito estável nas medições, oscilando muito próximos a $0,5 \mu \mathrm{m}$ com elevação dos valores medidos a partir do avanço de 0,12 $\mathrm{mm}$. Quando usinado em velocidade de $424 \mathrm{~m} \cdot \mathrm{min}^{-1}$, o material apresentou aumento na rugosidade, perdendo muita qualidade a partir de $0,12 \mathrm{~mm}$.

Observa-se no revestimento microcristalino (Fig. 5) estabilidade e pouca variação (pouco mais de $0,1 \mu \mathrm{m}$ ) em todo o comprimento do corpo de prova em todas as velocidades.

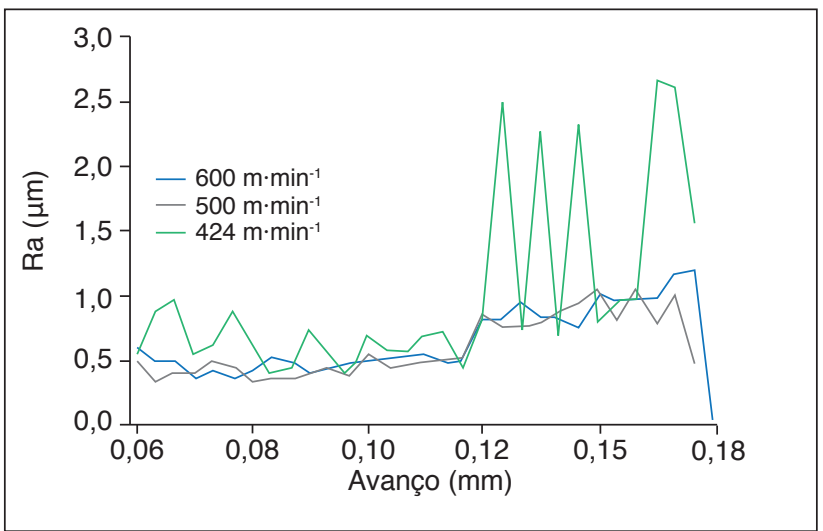

Figura 4: Comparativo de rugosidade entre velocidades no revestimento nanocristalino.

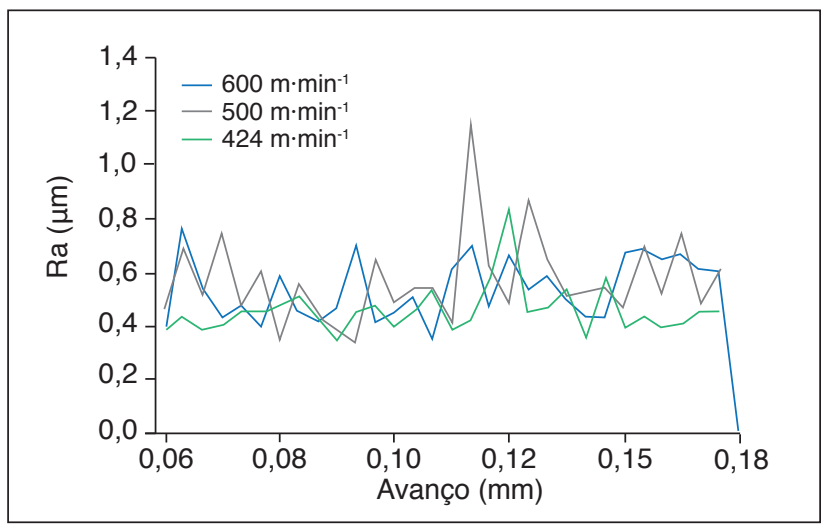

Figura 5: Comparativo de rugosidade entre velocidades no revestimento microcristalino.

\section{Cavacos}

A observação visual dos cavacos resultantes da usinagem em todos os experimentos de $1 \mathrm{~mm}$ (Figs. 6-8) mostra cavaco contínuo em forma de fita. Entretanto, os comprimentos e volume ocupado variaram em função da velocidade de corte aplicada, do diâmetro usinado e do tipo de ferramenta. A baixa relação entre a profundidade de usinagem e o raio de ponta da ferramenta favorece a formação de cavaco contínuo ${ }^{3}$. Observa-se em todas as condições que o volume dos cavacos é mais compacto em diâmetros menores de usinagem.

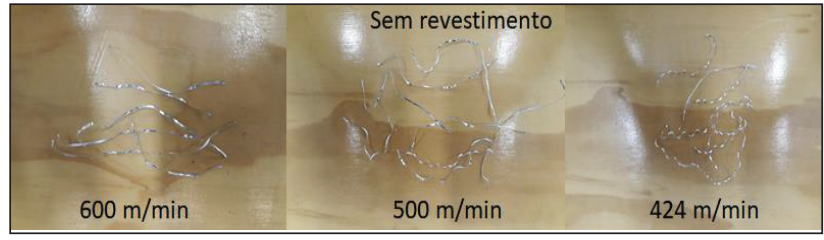

Figura 6: Cavacos gerados com inserto sem revestimento.

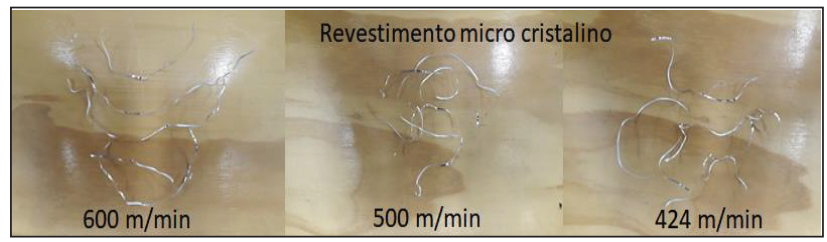

Figura 7: Cavacos gerados com inserto com revestimento microcristalino.

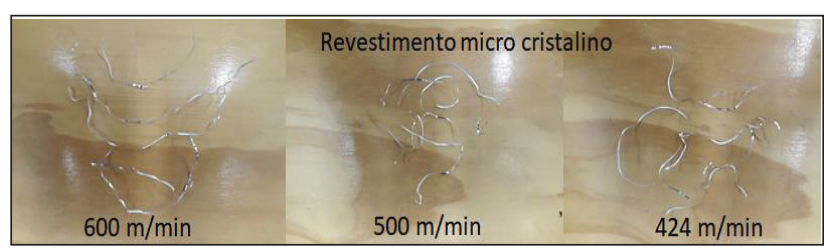

Figura 8: Cavacos gerados com inserto com revestimento nanocristalino.

\section{Desgaste dos insertos}

As investigações das propriedades químicas, estruturais e morfológicas dos filmes crescidos como monocamadas nano e microcristalinas deram-se por microscopia de força atômica (MFA), técnica de análise que consiste na varredura da superfície de uma amostra com uma sonda, a fim de obter sua imagem topográfica com resolução atômica ${ }^{15}$, e por microscopia eletrônica de varredura (MEV), que permite investigar a superfície de amostras com magnificações da ordem de centenas de milhares de vezes, visando identificação da morfologia de crescimento obtida e a correlação entre densidade de nucleação e os parâmetros de crescimento $^{16}$. Além disso, a técnica permite a realização de microanálises por espectroscopia de energia dispersiva (EDS, do inglês energy dispersive spectroscopy), que possibilita a identificação e o mapeamento dos elementos químicos presentes no material (Fig. 9).
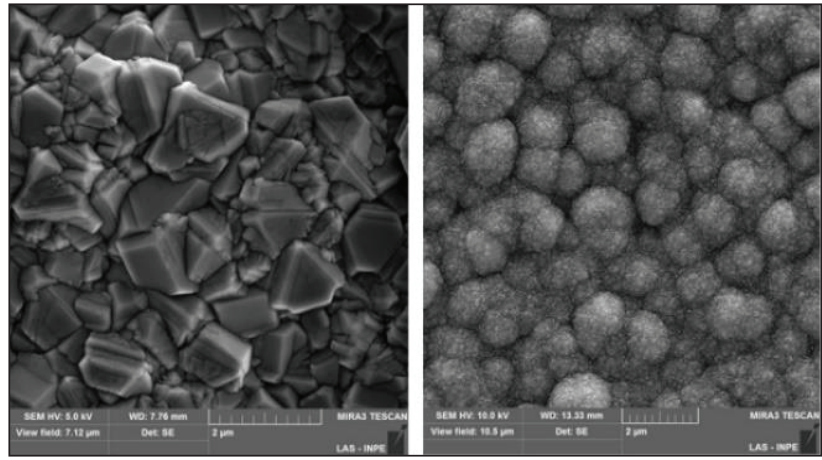

Figura 9: MEV dos filmes micro e nanocristalinos. 
A rugosidade do filme nanocristalino foi obtida através de MFA e está exposta na Tabela 4 .

Tabela 4: Rugosidade do inserto recoberto com diamante nanocristalino.

\begin{tabular}{|c|}
\hline Área da superfície \\
\hline $\mathbf{R q}$ \\
\hline Área da superfície projetada \\
\hline Diferença da área da superfície \\
\hline $\mathbf{R a}$ \\
\hline $\mathbf{R m a x}$ \\
\hline
\end{tabular}

$$
\begin{gathered}
10,5 \mu \mathrm{m}^{2} \\
39,1 \mathrm{~nm} \\
9,0 \mu \mathrm{m}^{2} \\
16,5 \% \\
31,8 \mathrm{~nm}
\end{gathered}
$$$$
300 \mathrm{~nm}
$$

Filmes compostos por grãos facetados de tamanho variando entre valores menores que $1,0 \mu \mathrm{m}$ foram obtidos, e podem ser observados na Fig. 10.

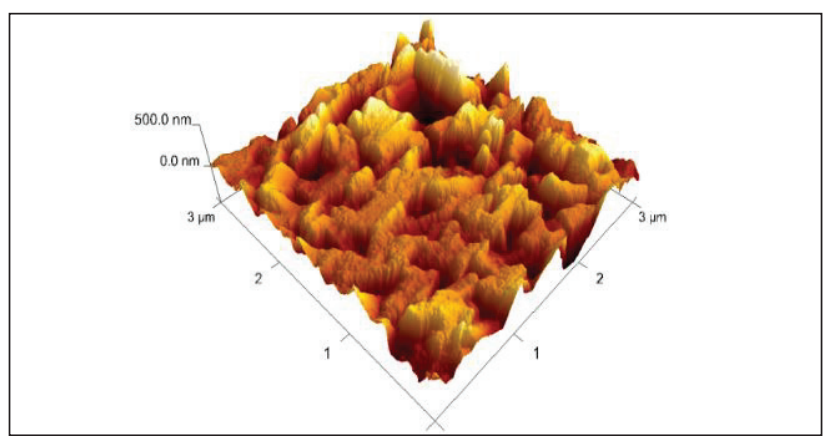

Figura 10: Imagem da rugosidade do filme nanocristalino medido por MFA.

A rugosidade do filme microcristalino está exposta na Tabela 5.

Tabela 5: Rugosidade do inserto recoberto com diamante microcristalino.

\begin{tabular}{|c|}
\hline Área da superfície \\
\hline $\mathbf{R q}$ \\
\hline Área da superfície projetada \\
\hline Diferença da área da superfície \\
\hline $\mathbf{R a}$ \\
\hline $\mathbf{R m a x}$ \\
\hline
\end{tabular}

$10,9 \mu \mathrm{m}^{2}$

$59,8 \mathrm{~nm}$

$9,0 \mu \mathrm{m}^{2}$

$21,0 \%$

$45,2 \mathrm{~nm}$

$515 \mathrm{~nm}$

Filmes compostos por grãos facetados de tamanho variando entre 1,0 e 1,5 $\mu \mathrm{m}$ foram obtidos e podem ser observados na Fig. 11.

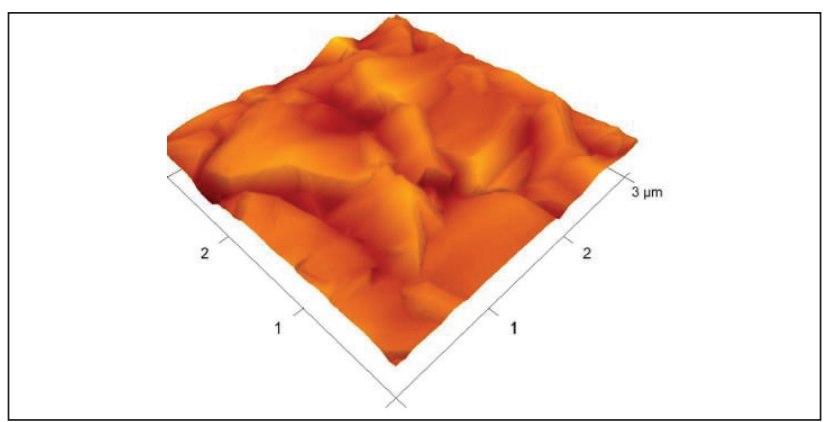

Figura 11: Rugosidade do inserto com diamante microdepositado medida por MFA.

\section{Filme microcristalino}

Com base nas análises EDS, pode-se observar que, para ambos os filmes, há depósito de alumínio na aresta de corte, porém, sem desgaste da aresta, e que os mecanismos de desgaste foram adesão e abrasão (Figs. 12 e 13).

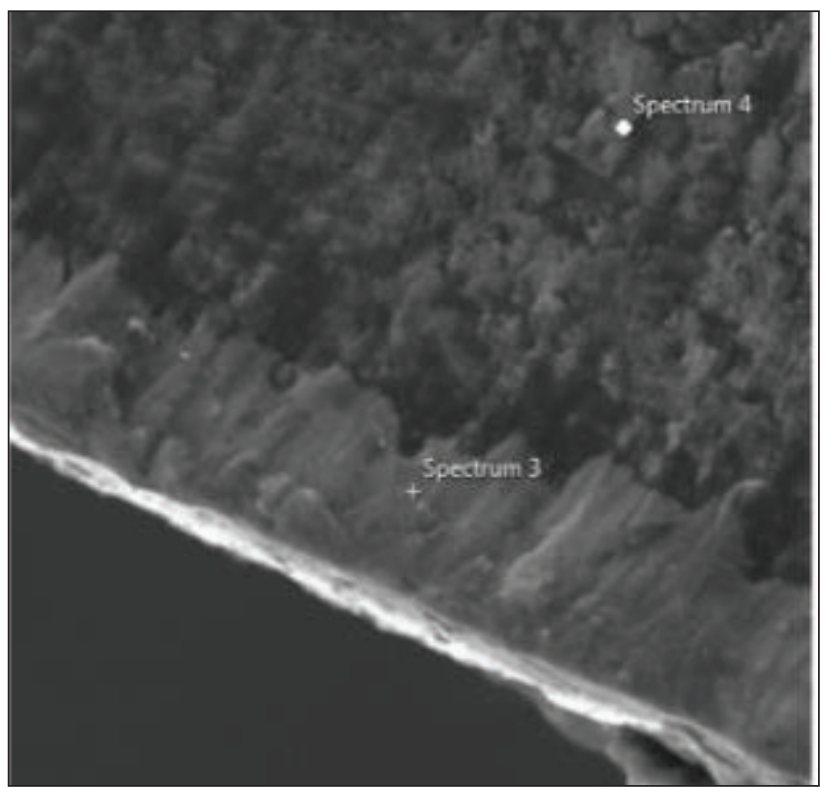

Figura 12: Pontos de leitura EDS em filme microcristalino.
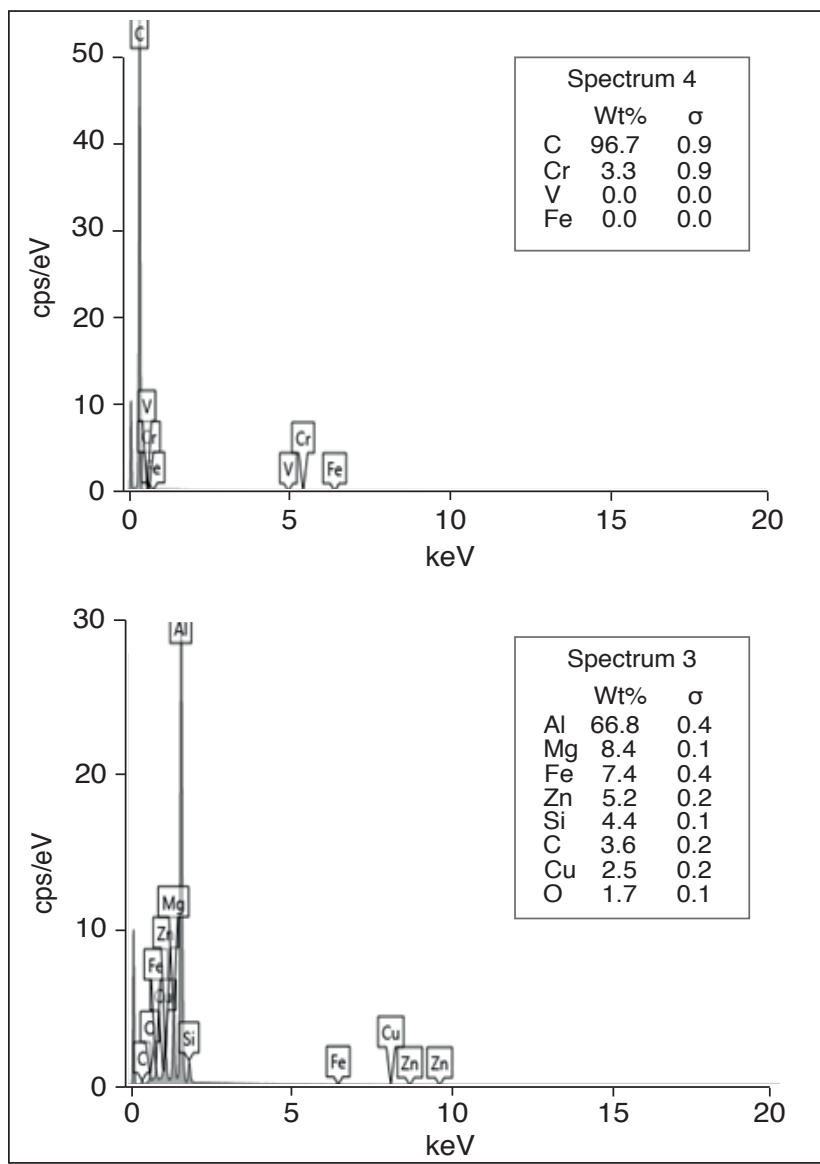

Figura 13: EDS em inserto com revestimento microcristalino. 
Acredita-se que as reentrâncias no revestimento de diamante CVD da ferramenta promova aderência do alumínio. O alumínio, uma vez aderido ao CVD, é ainda mais compactado e deformado pelas altas pressões de corte (Fig.14). A adesão entre o alumínio e o revestimento de diamante CVD pode se tornar tão forte que excede a adesão entre o revestimento de diamante e o substrato de metal duro, resultando em um potencial mecanismo para a delaminação do diamante ${ }^{17}$.
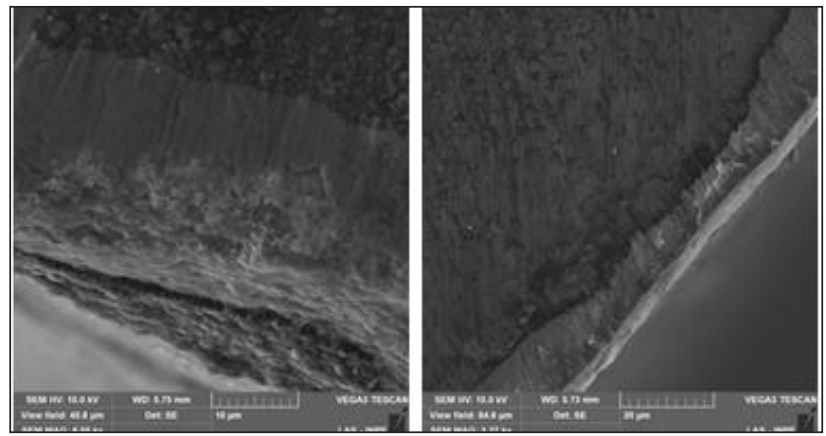

Figura 14: MEV da aresta de corte do inserto com filme nanocristalino.

A análise EDS (Figs. 15 e 16) para o filme nanocristalino mostra que há um depósito maior de alumínio do que no filme microcristalino, o que se deve ao perfil de cada filme (tamanho do grão, formato, rugosidade superficial).

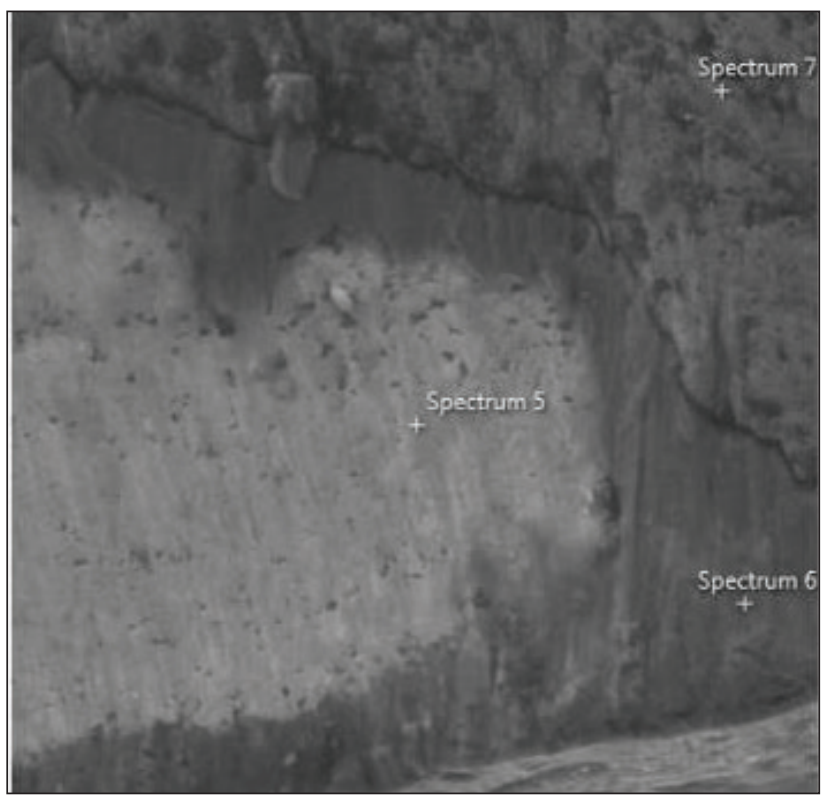

Figura 15: Pontos de leitura EDS em filme nanocristalino.

\section{CONCLUSÕES}

Em todos os casos, quando houve aumento do avanço, houve também aumento da rugosidade superficial, sendo que na ferramenta com revestimento nanocristalino esse aumento foi mais significativo. Pode-se concluir que o inserto microcristalino, embora apresente variação de rugosidade maior do que o nanocristalino, tem performance mais estável em todas as

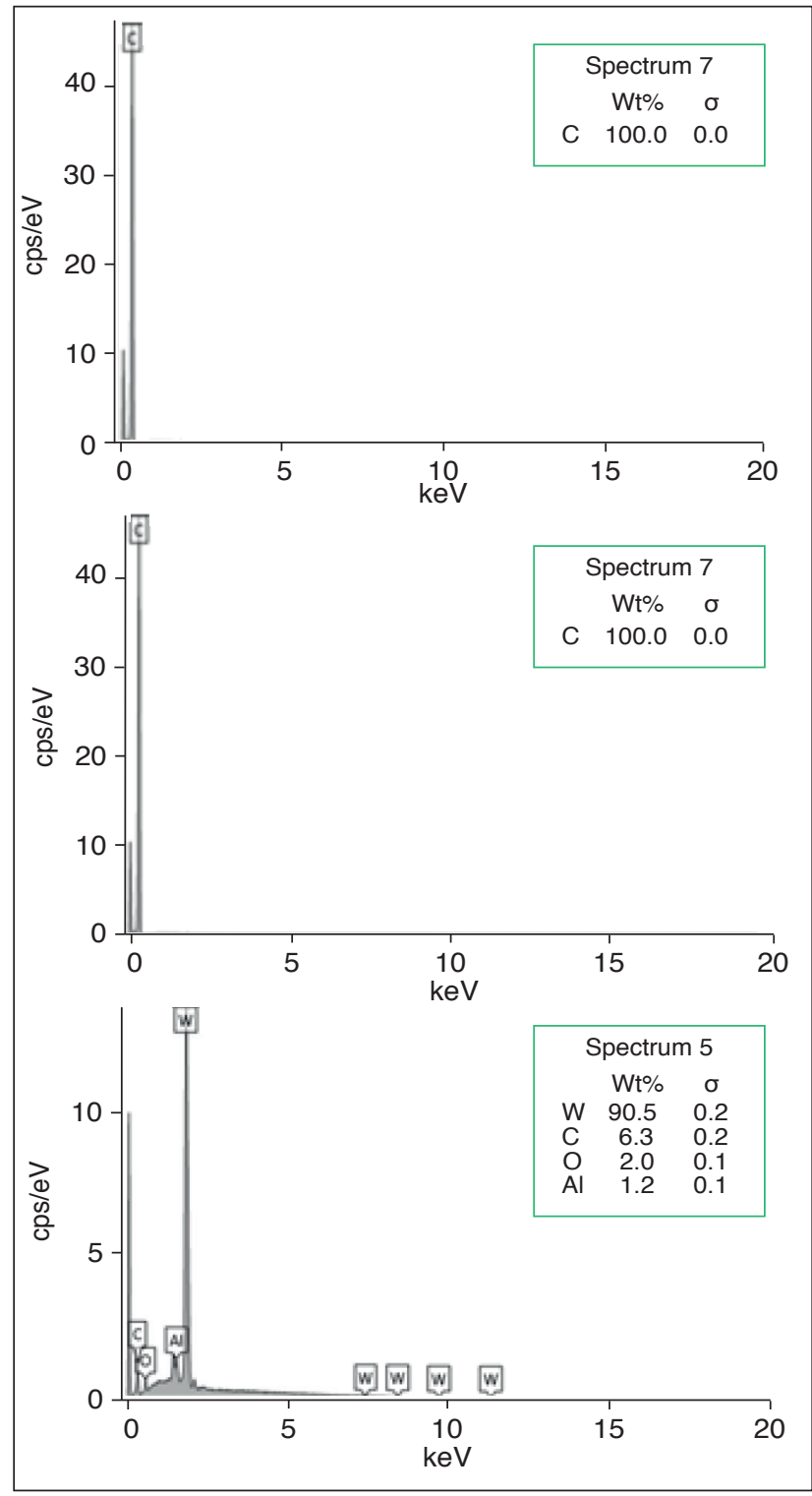

Figura 16: EDS em inserto com revestimento nanocristalino.

condições com valores entre 0,4 e $0,6 \mu \mathrm{m}$, estando o revestimento nanocristalino mais estável para velocidades maiores (acima de $500 \mathrm{~m} \cdot \mathrm{min}^{-1}$ ) com rugosidade estável em $0,5 \mu \mathrm{m}$.

- Os filmes não sofreram desgaste significativo por abrasão, porém houve acúmulo de alumínio em sua aresta de corte.

- Tendo em vista os resultados das análises, pode-se concluir que a escolha de um ou outro filme bem como a não utilização de inserto revestido deve levar em conta a relação custo-benefício e os parâmetros de acabamento envolvidos em cada trabalho a ser realizado.

\section{AGRADECIMENTOS}

O presente trabalho foi realizado com apoio da Coordenação de Aperfeiçoamento de Pessoal de Nível Superior - Brasil (CAPES) Código de Financiamento 001 


\section{REFERÊNCIAS}

1. Dursun T, Soutis C. Recent developments in advanced aircraft aluminium alloys. Mater Des. 2014;56:862-71. https://doi. org/10.1016/j.matdes.2013.12.002

2. Associação Brasileira de Alumínio. Anuário Estatístico ABAL 2017. São Paulo (SP): ABAL; 2017.

3. Dubar M, Dubois A, Dubar L. Wear analysis of tools in cold forging: PVD versus CVD TiN coatings. Wear. 2005;259(712):1109-16. https://doi.org/10.1016/j.wear.2005.01.006

4. Derflinger $\mathrm{V}$, Brändle $\mathrm{H}$, Zimmermann $\mathrm{H}$. New hard/lubricant coating for dry machining. Surf Coat Technol. 1999;113(3):28692. https://doi.org/10.1016/S0257-8972(99)00004-3

5. Gomez H, Durham D, Xiao X, Lukitsch M, Lu P, Chou K, Sachdev A, Kumar A. Adhesion analysis and dry machining performance of CVD diamond coatings deposited on surface modified WCCo turning inserts. J Mater Process Technol. 2012;212(2):523-33. https://doi.org/10.1016/j.jmatprotec.2011.10.020

6. Groover MP. Fundamentals of Modern Manufacturing: Materials, Processes, and Systems. 4 ed. Hoboken (NJ): John Wiley \& Sons; 2010.

7. Sun FH, Zhang ZM, Chen M, Shen HS. Improvement of adhesive strength and surface roughness of diamond films on Co-cemented tungsten carbide tools. Diamond Relat Mater. 2003;12(3-7):7118. https://doi.org/10.1016/S0925-9635(02)00345-X

8. Shen B, Sun F. Deposition and friction properties of ultra-smooth composite diamond films on Co-cemented tungsten carbide substrates. Diamond Relat Mater. 2009;18(2-3):238-43. https:// doi.org/10.1016/j.diamond.2008.10.053
9. Coppermetal Aços e Metais. Itda. São Paulo (SP): Certificado de qualidade 17304 Alumínio 7075-T651.

10. Cremer R, Mertens $R$, Neuschütz $D$, Lemmer $O$, Frank $M$, Leyendecker T. Formation of intermetallic cobalt phases in the near surface region of cemented carbides for improved diamond layer deposition. Thin Solid Films. 1999;355-356:127-33. https:// doi.org/10.1016/S0040-6090(99)00508-8

11. Wang $T$, Xiang $L$, Shi $W$, Jiang X. Deposition of diamond//B-SiC/ cobalt silicide composite interlayers to improve adhesion of diamond coating on WC-Co substrates by DC-Plasma Assisted HFCVD. Surf Coat Technol. 2011;205(8-9):3027-34. https://doi. org/10.1016/j.surfcoat.2010.11.014

12. Silva Neto JV, Rodríguez LAA, Fraga MA, Contin A, Campos RA, Corat EJ, Airoldi VJT. Preparação do substrato de WC-Co e condições de deposição. Rev Bras Apl Vac. 2016;35(1):53-7. https://doi.org/10.17563/rbav.v35i1.1015

13. Silva Neto JV. Crescimento de filmes de diamante CVD de alta aderência e baixas tensões residuais sobre substratos de WCCO [Dissertação de Mestrado]. São José dos Campos (SP). Instituto Nacional de Pesquisas Espaciais - INPE; 2018.

14. Trent E, Wright P. Metal Cutting. 4 ed. Boston (MA): ButterwortHeinemann, 2000.

15. Rugar D, Hansma P. Atomic Force Microscopy. Phys Today. 1990;43(10):23-30. https://doi.org/10.1063/1.881238

16. Diniz AE, Marcondez FC, Coppini NL. Tecnologia de usinagem dos materiais. 8 ed. São Paulo (SP): Artliber; 2013.

17. Andrewes CJE, Feng H-Y, Lau WM. Machining of an aluminum/ SiC composite using diamond inserts. J Mater Process Technol. 2000;102(1-3):25-9. https://doi.org/10.1016/S09240136(00)00425-8 